\title{
The effect of low dose Aspirin on age-related hearing loss
}

\begin{abstract}
Objective: The aim of the research was to find out if administration of low dose Aspirin $(81 \mathrm{mg})$ had a protective impact against age related hearing loss.

Methods: We performed a retrospective non-interventional study of all patients at King Abdulaziz University hospital (KAUH) who had age related hearing loss. A total of 31 patients were collected, looking at different variables among them. A telephone questionnaire was included to collect missing information. We further classified them based on the use of low dose Aspirin, and degree of hearing loss by looking at their last audiometric data. Lastly we selected patients who had multiple audiometric data to see the effect of low dose aspirin on their hearing.
\end{abstract}

Results: A total of 31 patients with age related hearing loss were included in this study, 13 out of them were males, from which 7 were on low dose aspirin, and 18 were females, from which 8 were on low dose Aspirin. With classification of their degree of hearing loss, the majority of patients on low dose aspirin had mild to moderate hearing loss, while the majority of patients not on Aspirin had normal to mild degree of hearing loss. When we looked at the differences between intensities of each frequency of multiple readings of 16 patients, which showed that $50 \%$ of them were on low dose aspirin and all of them showed preservation in their hearing. However, patients who were not on Aspirin, $31.3 \%$ of them showed preservation in their hearing and $18.8 \%$ did not have any effect on hearing preservation.

Conclusion: Low dose aspirin did not show hearing protection when we looked at one audiometric data done for patients with age related hearing loss. However, when multiple audiometric data were compared among patients depending whether they are using low dose Aspirin or not, there was a positive impact of low dose aspirin on the preservation of hearing. Studies with larger sample sizes are needed to support our observation.
Volume 10 Issue 5 - 2018

Talal Al-Khatib,' Khalid Alqarni, ${ }^{2}$ Mooataz Aashi,' Sarah Alsebai,' Mazin Merdad,' Hani Marzouki,'

'Department of Otolaryngology-Head Neck Surgery, King Abdulaziz University, Saudi Arabia

${ }^{2}$ Medical House Officers, Faculty of Medicine, King Abdulaziz University, Saudi Arabia

Correspondence: Talal Alkhatib, Department of Otolaryngology-Head Neck Surgery, Faculty of Medicine, King Abdulaziz University, P.o Box 80215, Jeddah 21589, Saudi Arabia, Tel 00966550574333, Email talkhatib@gmail.com

Received: July 16, 2018 | Published: September 10, 2018

\section{Background}

Salicylate, which is the active constituent of Aspirin, is usually used as an anti-inflammatory, analgesic and antipyretic drug. Moreover, people at high risk of heart attack take low dose of Aspirin daily ${ }^{1}$ as a mean of secondary prevention. ${ }^{2}$ With regard to its protective effects, Aspirin as an antioxidant appears to be neuro- and otoprotective. ${ }^{3}$ However, studies show that administration of high doses Aspirin (325 $\mathrm{mg}$ ) had an ototoxic effect leading to hearing loss. ${ }^{4}$ On the other hand, studies have shown that co-administration of Aspirin with gentamicin, which is considered to be ototoxic, had a protective effect against hearing loss,$^{5}$ also animal studies have shown that NSAIDs in low doses can protect against cochlear injury due to noise and aging, but the effect on human is yet to be determined. ${ }^{6}$ In our study, we aimed to find out if administration of low dose Aspirin $(81 \mathrm{mg})$ had a protective impact against hearing loss due to aging.

\section{Methodology}

This was a retrospective non-interventional study of all patients who had age related hearing loss in the past five years. The study was approved by the institutional review board (IRB). A total of 31 patients who had age related hearing loss, were included and their data were gained from the medical records. The following data was extracted from the records, demographic data, use, duration\& dose of Aspirin, other ear diseases, medications, and chronic illnesses.
Also we included a telephone questionnaire to collect the missed data from medical records, including their medical condition, if they were exposed to loud sounds for long periods at some points of their life and if they ever used any ototoxic medications. The research team took the last reading of the Pure Tone Audiometry (PTA) readings from all 31 patients, and multiple readings of different dates of the PTA data from 16 of them.

Classification of the degree of hearing loss was done according to Jerger's classification, ${ }^{7}$ and since there was asymmetry in hearing in most patients, the correlation was made between each ear and the use of aspirin. In the latter group, the average difference between the intensity (in $\mathrm{dBnHL}$ ) of each frequency of PTA readings depending on the duration of Aspirin use was calculated. Data entry was done using Microsoft Excel while analysis was done using SPSS version 19. Student $t$ test and Pearson's Chi square were used to test associations where appropriate.

\section{Results}

The study looked at the effect of low dose aspirin on the PTA readings of 31 patients who had age-related hearing loss during the last 5 year-period under review. $41.9 \%$ of them were male, and $58.1 \%$ were female.

Almost half of our patients were on low dose aspirin (48.4\%). Some patients, who were on low dose aspirin, had a mild to moderate 
degree of hearing loss $(9.7 \%$ in the right ear, $12.9 \%$ in the left ear).

On the other hand, patients who weren't on low dose Aspirin had normal to mild degree of hearing loss $(12.9 \%$ in the right ear, $16.1 \%$ in the left ear) (Table 1). The difference statistically was not significant ( $p=0.295$ and $p=0.503$ respectively).

Table 1 shows that most patients who were on low dose aspirin, had a mild to moderate degree of hearing loss in left ear(12.9\%), on the other hand $16,1 \%$ of patients who weren't on Aspirin had normal to mild degree of hearing loss. In the right ear, $9.7 \%$ of patients on low dose Aspirin had mild to moderate degree of hearing loss, and 12,9\% of patients who weren't on Aspirin had normal to mild degree of hearing loss. The difference statistically was not significant $(\mathrm{p}=0.295)$ $(\mathrm{p}=0.503)$ respectively

The difference between threshold intensity levels of frequencies $250,500,1000,2000$, and 4000 were calculated in 16 patients with multiple PTA readings over the years, $50 \%$ of them were on Aspirin and all of them showed preservative effect on the hearing (Table 2). $50 \%$ of patients were not on aspirin, $31.3 \%$ of them showed unchanged hearing levels, while $18.8 \%$ had deterioration of hearing. Though the difference statistically was not significant $(\mathrm{P}=0.1)$.

Table I The Effect of low Dose Aspirin on Degree of Hearing loss in single reading

\begin{tabular}{|c|c|c|c|c|c|c|c|}
\hline \multirow{2}{*}{$\begin{array}{l}\text { Degree of hearing loss in } \\
\text { Right ear }\end{array}$} & \multicolumn{2}{|c|}{ Use of Aspirin } & \multirow{2}{*}{ Total } & \multirow{2}{*}{$\begin{array}{l}\text { Degree of hearing loss in } \\
\text { Left ear }\end{array}$} & \multicolumn{2}{|c|}{ Use of Aspirin } & \multirow{2}{*}{ Total } \\
\hline & Yes & No & & & Yes & No & \\
\hline Normal to mild & $2(6.5 \%)$ & $4(12.9 \%)$ & 6 & Normal to mild & $\mathrm{I}(3.2 \%)$ & $5(16.1 \%)$ & 6 \\
\hline Normal to Profound & $\mathrm{I}(3.2 \%)$ & 0 & I & Normal to Moderate & $\mathrm{I}(3.2 \%)$ & $2(6.5 \%)$ & 3 \\
\hline Mild to Moderate & $3(9.7 \%)$ & $\mathrm{I}(3.2 \%)$ & 4 & Normal to Sever & $3(9.7 \%)$ & 0 & 3 \\
\hline Mild to Sever & $2(6.5 \%)$ & $\mathrm{I}(3.2 \%)$ & 3 & Mild to Moderate & $4(12.9 \%)$ & $2(6.5 \%)$ & 6 \\
\hline Mild to Profound & $2(6.5 \%)$ & 0 & 2 & Mild to Sever & $\mathrm{I}(3.2 \%)$ & $\mathrm{I}(3.2 \%)$ & 2 \\
\hline Moderate to Sever & 0 & $\mathrm{I}(3.2 \%)$ & 1 & Mild to Profound & $\mathrm{I}(3.2 \%)$ & $2(6.5 \%)$ & 3 \\
\hline Moderate to Profound & 0 & $\mathrm{I}(3.2 \%)$ & I & Moderate to Sever & $\mathrm{I}(3.2 \%)$ & $\mathrm{I}(3.2 \%)$ & 2 \\
\hline Sever to Profound & $\mathrm{I}(3.2 \%)$ & 0 & I & Moderate to Profound & $\mathrm{I}(3.2 \%)$ & $2(6.5 \%)$ & 3 \\
\hline Normal & $2(6.5 \%)$ & $2(6.5 \%)$ & 4 & Sever to Profound & $\mathrm{I}(3.2 \%)$ & 0 & I \\
\hline Mild & $\mathrm{I}(3.2 \%)$ & $3(9.7 \%)$ & 4 & Mild & $\mathrm{I}(3.2 \%)$ & $\mathrm{I}(3.2 \%)$ & 2 \\
\hline Moderate & $\mathrm{I}(3.2 \%)$ & 0 & 1 & & & & \\
\hline Profound & 0 & $3(9,7 \%)$ & 3 & Total & $15(48.4 \%)$ & $16(51.6 \%)$ & 31 \\
\hline Total & I5(48.4\%) & $16(51.6 \%)$ & 31 & & & & \\
\hline
\end{tabular}

Table I shows that most patients who were on low dose aspirin, had a mild to moderate degree of hearing loss in left ear(I2.9\%), on the other hand I6, I\% of patients who weren't on Aspirin had normal to mild degree of hearing loss. In the right ear, $9.7 \%$ of patients on low dose Aspirin had mild to moderate degree of hearing loss, and $12,9 \%$ of patients who weren't on Aspirin had normal to mild degree of hearing loss. The difference statistically was not significant $(p=0.295)(p=0.503)$ respectively

Table 2 The effect of low dose aspirin on degree of hearing loss in multiple reading

\begin{tabular}{|c|c|c|c|}
\hline \multirow[t]{2}{*}{ Use of aspirin } & \multicolumn{2}{|c|}{ Effect on hearing loss } & \multirow[t]{2}{*}{ Total } \\
\hline & Positive effect & No effect & \\
\hline Yes & $8(50 \%)$ & 0 & 8 \\
\hline No & $5(31,3 \%)$ & $3(18,8 \%)$ & 8 \\
\hline Total & $\mid 3(8 \mid, 3 \%)$ & $3(18,8 \%)$ & $16(100 \%)$ \\
\hline
\end{tabular}

\section{Discussion}

Aspirin is widely used as an anti-inflammatory, analgesic, and antipyretic drug. Moreover, it's been used daily with low doses as means of secondary prevention for people at high risk of developing heart attack. ${ }^{1}$ However, studies showed that administration of high dose aspirin $(325 \mathrm{mg})$ had an ototoxic effect including hearing loss. ${ }^{4}$

Animal studies have shown that NSAIDs in low doses can protect against cochlear injury due to noise and aging, but the effect on human is yet to be determined. ${ }^{6}$
A total of 31 patients who had age related hearing loss, with the exclusion of drug induced and noise induced hearing loss were included in the study. Thirteen $(41.9 \%)$ of them were male, from which $7(22.6 \%)$ were on low dose aspirin and $18(58.1 \%)$ of them were female, from which $8(25.8 \%)$ were on low dose aspirin. When we looked at the last audiometric data done for patients with age related hearing loss some patients, who were on low dose aspirin, had a mild to moderate degree of hearing loss $(9.7 \%$ in the right ear, $12.9 \%$ in the left ear). On the other hand, patients who weren't on low dose Aspirin had normal to mild degree of hearing loss $(12.9 \%$ in the 
right ear, $16.1 \%$ in the left ear) (Table 1 ). The difference statistically was not significant $(\mathrm{p}=0.295$ and $\mathrm{p}=0.503$ respectively). However, when the average difference between intensities of each frequency was calculated on a selected sample of 16 patients (who had age related hearing loss, with multiple audiometric data over the last 5 years) a difference in hearing preservation was identified. Incidentally, eight of those patients $(50 \%)$ were on low dose Aspirin and $8(50 \%)$ weren't on low dose aspirin. All the 8 patients who were on low dose aspirin showed preservation in their hearing. What we meant by hearing preservation is no change or deterioration in hearing level. In contrast, patients who weren't on aspirin, 5 of them [31.3\%] had no hearing change while 3 of them [18.8\%] had deterioration in their hearing. Our results support the study that concluded that "aspirin as an antioxidant appears to be neuro- and oto-protective". ${ }^{3}$ On the other hand, our results oppose a Korean study, which showed few reports of salicylate-induced ototoxicity, and the majority was caused by a low dose of aspirin. ${ }^{8}$

\section{Conclusion}

Low dose aspirin did not show hearing protection when we looked at one (the last) audiometric data done for patients with age related hearing loss. However, when multiple audiometric data were compared among patients depending whether they are using low dose Aspirin or not, there was a positive impact of low dose aspirin on the preservation of hearing. Studies with larger sample sizes are needed to support our observation.

\section{Acknowledgments}

No financial support or grants.

\section{Conflict of interest}

All the authors have no conflict of interest.

\section{References}

1. Ridker PM, Cook NR, Lee IM, et al. A randomized trial of low-dose aspirin in the primary prevention of cardiovascular disease in women. N Engl J Med. 2005;352(13):1293-304.

2. Pietri P, Vlachopoulos C, Terentes-Printzios D, et al. Beneficial effects of low dose aspirinon aortic stiffness in hypertensive patients. Vasc Med. 2014;19(6):452-457.

3. Deng L, Ding D, Su J, et al. Salicylate selectively kills cochlear spiral ganglion neurons by paradoxically up-regulating superoxide. Neurotox Res. 2013;24(3):307-319.

4. Sheppard A, Hayes SH, Chen GD, et al. Review of salicylate-induced hearing loss, neurotoxicity, tinnitus and neuropathophysiology. Acta Otorhinolaryngol Ital. 2014;34(2):79-93.

5. Kyle ME, Wang JC, Shin JJ. Ubiquitous Aspirin: A Systematic Review of Its Impact on Sensorineural Hearing Loss. Otolaryngol Head Neck Surg. 2015;152(1):23-41.

6. Hoshino T, Tabuchi K, Hara A. Effects of NSAIDs on the inner ear: Possible involvement in cochlear protection. Pharmaceuticals (Basel) 2010;3(5):1286-1295.

7. Hayes D, Jerger J. The Effect of Degree of Hearing Loss on Diagnostic Test Strategy Report of a Case. Archives of Otolaryngollogy. 1980;106(5):266-268.

8. Kim SM, Jo JM, Baek MJ, et al. A Case of Bilateral Sudden Hearing Loss and Tinnitus after Salicylate Intoxication. Korean $J$ Audiol. 2013;17(1): 23-26. 